\title{
Plant Phenotypic and Transcriptional Changes Induced by Volatiles from the Fungal Root Pathogen Rhizoctonia solani
}

\author{
Viviane Cordovez 1,2, Liesje Mommer ${ }^{3}$, Kay Moisan ${ }^{1,4}$, Dani Lucas-Barbosa ${ }^{4}$, \\ Ronald Pierik ${ }^{5}$, Roland Mumm ${ }^{6,7}$, Victor J. Carrion ${ }^{1}$ and Jos M. Raaijmakers ${ }^{1,8 *}$ \\ ${ }^{1}$ Department of Microbial Ecology, Netherlands Institute of Ecology (NIOO-KNAW), Wageningen, Netherlands, ${ }^{2}$ Laboratory \\ of Phytopathology, Wageningen University, Wageningen, Netherlands, ${ }^{3}$ Plant Ecology and Nature Conservation Group, \\ Wageningen University, Wageningen, Netherlands, ${ }^{4}$ Laboratory of Entomology, Wageningen University, Wageningen, \\ Netherlands, ${ }^{5}$ Plant Ecophysiology, Institute of Environmental Biology, Utrecht University, Utrecht, Netherlands, \\ ${ }^{6}$ Wageningen Plant Research, Business Unit Bioscience, Wageningen University and Research, Wageningen, Netherlands, \\ 7 Centre for Biosystems Genomics, Wageningen, Netherlands, ${ }^{8}$ Institute of Biology, Leiden University, Leiden, Netherlands
}

OPEN ACCESS

Edited by:

Jens Staal,

Ghent University, Belgium

Reviewed by:

Maria L. Pappas,

Democritus University of Thrace,

Greece

Kemal Kazan,

Commonwealth Scientific and Industrial Research Organisation

(CSIRO), Australia

*Correspondence:

Jos M. Raaijmakers

j.raaijmakers@nioo.knaw.nl

Specialty section: This article was submitted to Plant Microbe Interactions,

a section of the journal

Frontiers in Plant Science

Received: 16 May 2017

Accepted: 04 July 2017

Published: 21 July 2017

Citation:

Cordovez V, Mommer L, Moisan K, Lucas-Barbosa D, Pierik $R$,

Mumm R, Carrion VJ and

Raaijmakers JM (2017) Plant

Phenotypic and Transcriptional Changes Induced by Volatiles from the Fungal Root Pathogen

Rhizoctonia solani.

Front. Plant Sci. 8:1262.

doi: 10.3389/fpls.2017.01262
Beneficial soil microorganisms can affect plant growth and resistance by the production of volatile organic compounds (VOCs). Yet, little is known on how VOCs from soil-borne plant pathogens affect plant growth and resistance. Here we show that VOCs released from mycelium and sclerotia of the fungal root pathogen Rhizoctonia solani enhance growth and accelerate development of Arabidopsis thaliana. Seedlings briefly exposed to the fungal VOCs showed similar phenotypes, suggesting that enhanced biomass and accelerated development are primed already at early developmental stages. Fungal VOCs did not affect plant resistance to infection by the VOC-producing pathogen itself but reduced aboveground resistance to the herbivore Mamestra brassicae. Transcriptomics of $A$. thaliana revealed that genes involved in auxin signaling were up-regulated, whereas ethylene and jasmonic acid signaling pathways were downregulated by fungal VOCs. Mutants disrupted in these pathways showed similar VOC-mediated growth responses as the wild-type $A$. thaliana, suggesting that other yet unknown pathways play a more prominent role. We postulate that $R$. solani uses VOCs to predispose plants for infection from a distance by altering root architecture and enhancing root biomass. Alternatively, plants may use enhanced root growth upon fungal VOC perception to sacrifice part of the root biomass and accelerate development and reproduction to survive infection.

Keywords: fungal volatiles, plant growth promotion, plant resistance, plant transcriptome, auxin

\section{INTRODUCTION}

Plants interact with a multitude of (micro)organisms, including beneficial symbionts, fungal pathogens and insects. In the chemical interplay between microorganisms and plants, a wide range of compounds play a significant role. In this context, specific emphasis is given to volatile organic compounds (VOCs). This chemically diverse group of compounds can travel longer distances than other metabolites, facilitating a multitude of interactions with other organisms both below- and aboveground (Schulz and Dickschat, 2007; Das et al., 2012; Junker and Tholl, 2013). For example, 
during insect herbivore attack, plants trigger the emission of VOCs to attract natural enemies of the herbivores and to elicit resistance mechanisms in neighboring plants (Paré and Tumlinson, 1999; Dicke and Loreto, 2010; Mumm and Dicke, 2010). In addition, plants use VOCs during resource limitation to detect the presence of proximate competitors, such as neighboring plants, and to inhibit the competitors' growth and development (Kegge and Pierik, 2010).

Plants also perceive VOCs emitted by other (micro)organisms present in their environment. It is now well established that VOCs emitted by beneficial soil microorganisms mediate, from a distance, interactions with plants and other microorganisms (Bailly and Weisskopf, 2012; Effmert et al., 2012; Bitas et al., 2013; Schmidt et al., 2015). VOCs emitted by plant-associated bacteria have been reported to promote plant growth, to induce plant systemic resistance, and to affect motility and antibiotic resistance in other bacteria (Ryu et al., 2003, 2004; Lee et al., 2012; D'Alessandro et al., 2014; Park et al., 2015). Fungal VOCs are also widespread in nature, but less well studied than bacterial VOCs. To date, approximately 250 fungal VOCs have been described, including acids, alcohols, aldehydes, esters, short-chain fatty acids, lipid oxides, terpenes and phenolics (Morath et al., 2012; Roze et al., 2012). The most well-known fungal VOC is 1-octen3-ol, also referred to as the "mushroom smell," which functions as a developmental signal for several fungal species (Chitarra et al., 2004; Herrero-Garcia et al., 2011).

To date, information on the ecological functions of fungal VOCs is limited and fragmented, in particular for VOCs produced by soil-borne plant pathogenic fungi. Hung et al. (2013) showed that VOCs emitted by the mycoparasitic fungus Trichoderma viride increased biomass and chlorophyll content of Arabidopsis thaliana seedlings. In contrast, Kottb et al. (2015) showed that VOCs emitted by T. asperellum inhibited plant growth and increased the levels of the plant hormones salicylic and abscisic acid, resulting in enhanced resistance against pathogenic fungi. Bitas et al. (2015) further showed that VOCs emitted by Fusarium oxysporum promoted the growth of A. thaliana and Nicotiana tabacum, and affected auxin transport and signaling. A more recent study showed that VOCs emitted by Alternaria alternata enhanced growth, early flowering and photosynthesis rates of $A$. thaliana, maize and pepper by affecting the levels of plastidic cytokinin (Sanchez-Lopez et al., 2016). Collectively, these few studies suggest that VOCs from plant-associated fungi may modulate the trade-off between plant growth, development and resistance. However, the natural functions of fungal VOCs as well as the underlying molecular mechanisms induced in plants remain largely unknown.

Here, we investigated the short- and long-term effects of VOCs emitted by the soil-borne plant pathogenic fungus Rhizoctonia solani on growth, development and resistance of $A$. thaliana. We exposed seeds and seedlings to the fungal VOCs and investigated their impact on seed germination, shoot and root biomass, and resistance belowground to the VOC-producing fungus as well as aboveground to the generalist herbivore Mamestra brassicae. As a first step to understand the underlying molecular mechanisms, we performed genome-wide transcriptome analysis of $A$. thaliana seedlings exposed to $R$. solani VOCs and subsequently tested a range of $A$. thaliana mutants disrupted in genes involved in ethylene and auxin pathways. The putative natural functions of the VOCs in pathogen-plant interactions are discussed.

\section{MATERIALS AND METHODS}

\section{Plants, Fungi, and Insect}

Arabidopsis thaliana wild-type Col-0 was obtained from the collection of the Department of Phytopathology at Wageningen University, the Netherlands. Other genotypes used were the ethylene-insensitive mutants etr1-4 (Chang et al., 1993) and ein 3 eil1 (Alonso et al., 2003), the auxin receptor mutant tirl afb1, the auxin biosynthesis mutant wei8 [similar to sav3, (Stepanova et al., 2008)], the PHYTOCHROME INTERACTING FACTOR (PIF) double mutant pif4 pif5 (Lorrain et al., 2008). For the negative PIF regulator (HFR1) both the knockout mutant $h f r 1-5$ (Sessa et al., 2005) and the stable overexpressor p35S:G-BH-03 (Galstyan et al., 2011) were used. Seeds were surface sterilized as previously described (van de Mortel et al., 2012) and kept in the dark at $4^{\circ} \mathrm{C}$ for three to 4 days before sowing. Plates and pots containing plants were grown in climate cabinets $\left(21^{\circ} \mathrm{C} ; 180 \mu \mathrm{mol} \mathrm{light} \mathrm{m}^{-2}\right.$ $\mathrm{s}^{-1}$ at plant level; $16 \mathrm{~h}: 8 \mathrm{~h}$, light : dark; $60-70 \%$ R.H.).

The fungus $R$. solani AG2-2 IIIB was obtained from the collection of the Sugar Beet Research Institute, Bergen op Zoom, the Netherlands. Fungal cultures were started with a mycelial plug (Ø $5 \mathrm{~mm}$ ) on $1 / 5^{\text {th }}$ strength Potato Dextrose Agar $\left(1 / 5^{\text {th }} \mathrm{PDA}\right.$, Oxoid) at $25^{\circ} \mathrm{C}$. Sclerotia were obtained from a fungal culture incubated on $1 / 5^{\text {th }} \mathrm{PDA}$ at $25^{\circ} \mathrm{C}$ for 3 weeks.

The generalist insect herbivore $M$. brassicae L. (Lepidoptera: Noctuidae; cabbage moth) was reared on Brassica oleracea L. var. gemmifera cv. Cyrus in a controlled growth chamber $\left(22 \pm 2{ }^{\circ} \mathrm{C}\right.$; 16 h: 8 h, light: dark; 40-50\% R.H.). Newly-emerged larvae were used in the experiments.

\section{Effects of Fungal Volatiles on Plant Growth and Development}

To investigate the effects of VOCs emitted by the fungal pathogen $R$. solani on the growth and development of A. thaliana, 7-dayold seedlings were exposed to the fungal VOCs. To physically separate the plants from the fungus, a three-compartment set-up was used (Figure 1A). Sterile A. thaliana seeds were sown on Petri dishes (Ø $90 \mathrm{~mm}$ ) containing $25 \mathrm{~mL}$ of half-strength Murashige and Skoog medium (Murashige and Skoog, 1962) supplemented with $5 \%$ sucrose $(0.5 \mathrm{xMS})$. These Petri dishes (without lids) were kept inside a larger Petri dish ( $\varnothing 145 \mathrm{~mm})$ which were sealed and kept in climate cabinets. After 7 days, seedlings were exposed to the fungal VOCs or to agar medium by introducing a small Petri dish ( $\varnothing 35 \mathrm{~mm}$ ) containing a 7-day-old fungal culture (referred to as VOC-exposed) or containing the agar medium or a soil-sand mixture only (control). Petri dishes (Ø $145 \mathrm{~mm}$ ) were re-sealed and kept in the climate cabinets for 2 weeks. For testing the effect of different substrates on VOC production by the fungus, five different media were used. The media tested included water-agar (HA), water-agar supplemented with sucrose (HS), water-agar supplemented with sucrose and yeast extract (HSY), malt-agar (Oxoid) (Ezra and Strobel, 2003) and $1 / 5^{\text {th }}$ strength 

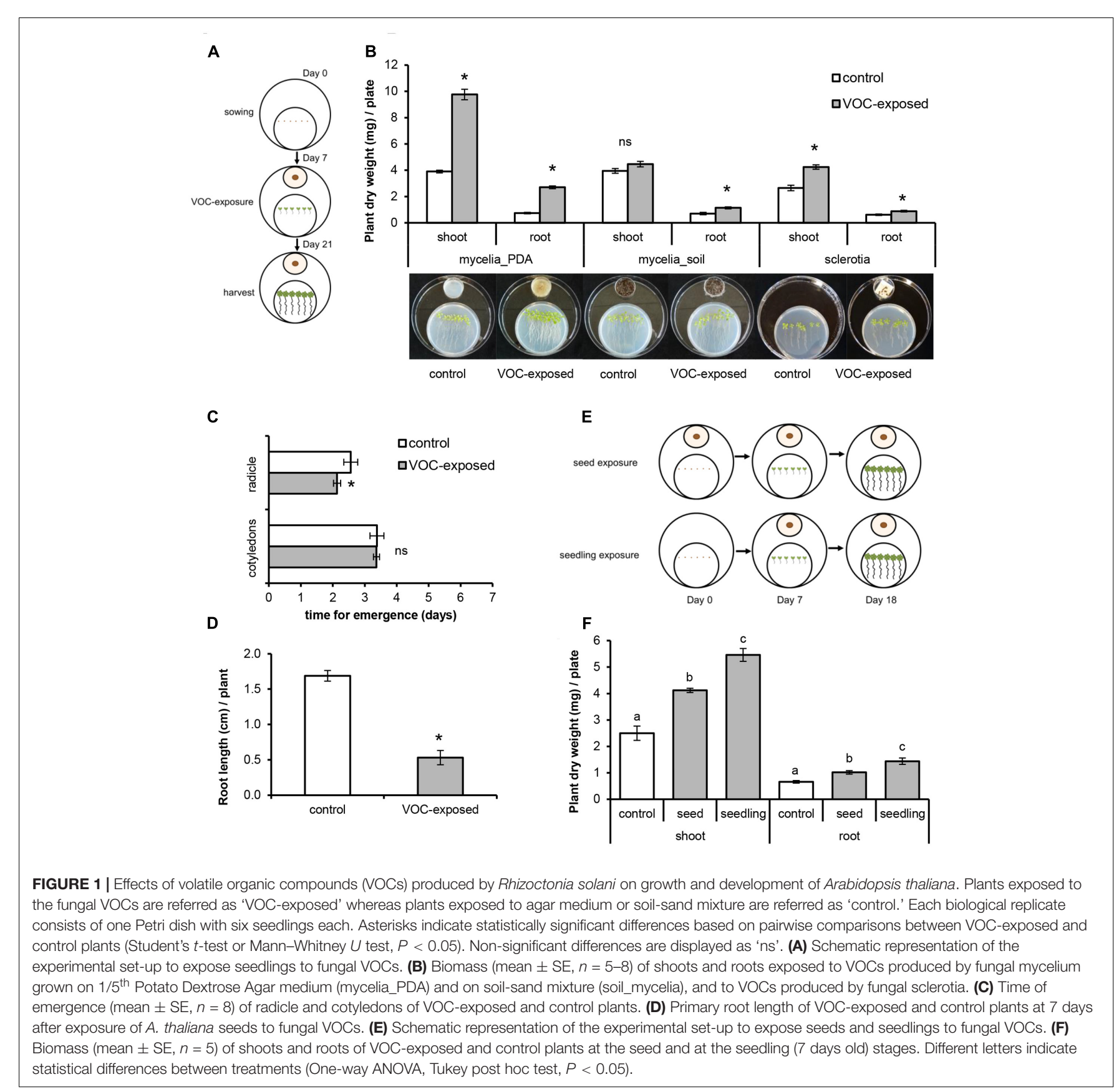

potato dextrose agar (1/5 th $^{\text {PDA }) . ~ A d d i t i o n a l l y, ~} R$. solani was grown in a soil-sand mixture. For that, a mycelium plug was added into $2.5 \mathrm{~g}$ of sterile soil-sand mixture $(12: 5 \mathrm{v} / \mathrm{v})$, previously sterilized by autoclaving for $20 \mathrm{~min}$ twice with $24 \mathrm{~h}$ interval. Fresh and dry weight (overnight incubation at $65^{\circ} \mathrm{C}$ ) of shoots and roots of VOC-exposed plants were determined and compared to control plants. For each treatment, five to six plants per plate were pooled and treated as a single biological replicate, and a total of five to eight biological replicates were used.

To test the effects of fungal VOCs on seed germination and emergence of the radicle and cotyledons, we used twocompartment Petri dishes (Ø $90 \mathrm{~mm}$ ). One compartment contained 0.5xMS medium and six seeds and the other contained a small Petri dish (Ø $35 \mathrm{~mm}$, without lid) with a 7-dayold fungal culture or $1 / 5^{\text {th }}$ PDA medium only (control). The two-compartment Petri dish was then closed and incubated as described previously. Seed germination and emergence of cotyledons and radicle were recorded daily. Length of primary root was measured after 7 days of exposure.

To study the effects of a short exposure to fungal VOCs on plant growth and development, 7-day-old A. thaliana seedlings grown in vitro were exposed to R. solani VOCs (VOC-exposed) or to the agar medium (control) for 1 week as described above and then transferred to pots containing a soil-sand mixture (1:1 
v/v) sterilized by autoclaving for 20 min twice with a $24 \mathrm{~h}$ interval (Figure 2A). Shoot fresh weight, length of floral stem, and number of flowers were determined 2 weeks after soil transplantation. To test if the observed effects of fungal VOCs on plant development could be transferred via the seeds to the next generation (transgenerational effect), 10 VOC-exposed and 10 control plants were kept for seed collection. Seeds were harvested, surface-sterilized, sown in Petri dishes $(\varnothing 90 \mathrm{~mm})$ containing $0.5 \mathrm{xMS}$ and grown for 2 weeks under controlled conditions. Fresh and dry weight (overnight incubation at $65^{\circ} \mathrm{C}$ ) of shoots and roots were determined and compared to control plants.

Statistical analyses were performed with IBM SPSS Statistics 23. Data were tested for equal variance using Levene's test and for normality using Shapiro-Wilk test at the 5\% significance level. Statistical differences were determined by pairwise comparisons of VOC-exposed and control plants with Student $t$-test (for independent samples) or One-way ANOVA followed by Tukey's honestly significant difference (HSD) post hoc test. MannWhitney $U$ test was used when assumptions of normality were not met.

\section{Effects of $\mathrm{CO}_{2}$ on Plant Growth}

$\mathrm{CO}_{2}$ concentrations are known to be increased in closed systems due to microbial catabolism which, in turn, could enhance photosynthetic efficiency and plant biomass. To investigate potential effects of increased levels of fungal-produced $\mathrm{CO}_{2}$ on plant growth, 7-day-old $A$. thaliana seedlings grown on square Petri dishes $(\varnothing 100 \mathrm{~mm}$ ) were exposed to ambient (400 ppm) and elevated (1330 ppm) $\mathrm{CO}_{2}$ levels in $22.4 \mathrm{~L}$ desiccators. A total of four to seven replicates were used per treatment. $\mathrm{CO}_{2}$ levels were manipulated through mass flow controllers mixing air and $\mathrm{CO}_{2}$ to the desired concentrations and monitored with infrared gas analyzers. Desiccators were kept at $20^{\circ} \mathrm{C}$; $180 \mu \mathrm{mol}$ light $\mathrm{m}^{-2} \mathrm{~s}^{-1}$ at plant level; $16 \mathrm{~h}: 8 \mathrm{~h}$, light: dark. Plant shoot and root biomass was determined after 7 days of exposure. Data were tested for equal variance using Levene's test and for normality using Shapiro-Wilk test at the 5\% significance level. Statistical differences were determined by pairwise comparisons of $\mathrm{CO}_{2}$ exposed and control plants with Student $t$-test (for independent samples) using IBM SPSS Statistics 23. Kruskal-Wallis test was used when assumptions of normality were not met.

\section{Collection and Analysis of Fungal Volatiles}

Since also ethylene, a gaseous hormone, is known to be produced by soil microorganisms and to play a role in plant growth and development, we determined the ethylene concentration emitted by $R$. solani. For that, a mycelial plug (Ø $5 \mathrm{~mm}$ ) was grown in sterile $10 \mathrm{~mL}$ glass vials containing $2.5 \mathrm{~mL}$ of $1 / 5^{\text {th }}$ PDA or $0.5 x M S$ media. Vials containing the media only were used as controls. All vials were closed with silicone/PTFE lids and incubated at $25^{\circ} \mathrm{C}$ for 10 days. Ethylene concentration was measured with a gas chromatograph (Syntech GC 955-100) equipped with a HayeSep 80/100 column and flame-ionization detector as previously described (Pierik et al., 2009). Measurements were performed using five replicates.
Statistical differences were determined by pairwise comparisons of treatment and control (medium only) with Mann-Whitney $U$ test using IBM SPSS Statistics 23.

To identify other VOCs produced by $R$. solani and potentially involved in the modulation of plant growth and development, fungal cultures were prepared as described for the ethylene measurements. Fungal VOCs were collected by a dynamic headspace system on stainless steel cartridges filled with $200 \mathrm{mg}$ Tenax TA (20/35; Camsco Inc.) as adsorbent. For this, a custom-made 'needle inlet' connected to a Tenax cartridge was penetrated through the septum in the lids. VOCs were collected on cartridges by sucking the air out of the vials with a flow of $40 \mathrm{~mL} \mathrm{~min}$ m $^{-1}$ for $5 \mathrm{~h}$. A second clean Tenax cartridge was placed similarly to clean the incoming air and to prevent an under pressure. Before thermodesorption, cartridges with the headspace samples were flushed with helium at $50 \mathrm{~mL} \mathrm{~min}^{-1}$ for $5 \mathrm{~min}$ to remove moisture and oxygen and after that analyzed by Thermodesorption Gas Chromatography-Mass Spectrometry (TDGC-MS). VOCs were thermally desorbed at $220^{\circ} \mathrm{C}$ for $7 \mathrm{~min}$ (Ultra, Markes Llantrisant, United Kingdom) with a helium flow of $30 \mathrm{~mL} \mathrm{~min}{ }^{-1}$. Analytes were focused at $4^{\circ} \mathrm{C}$ on a cooled trap (Unity, Markes, Llantrisant, United Kingdom) and were then transferred to the analytical column (ZB-5Msi, $30 \mathrm{~m}, 0.25 \mathrm{~mm}$ i.d., $1.0 \mu \mathrm{m}$ film thickness, Phenomenex, Torrance, CA, United States) by rapid heating of the cold trap to $260^{\circ} \mathrm{C}$ for $4 \mathrm{~min}$ using

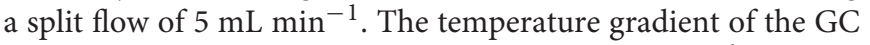

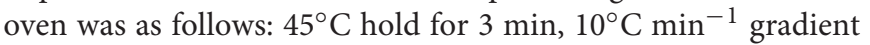
to $280^{\circ} \mathrm{C}$, with a $2 \mathrm{~min}$ hold, at a constant gas flow of $1 \mathrm{~mL}$ $\mathrm{min}^{-1}$. Mass spectra were acquired by electron impact ionization $(70 \mathrm{eV})$ with a scanning from $\mathrm{m} / \mathrm{z} 35-400$ with a scan rate of 5 scans s $^{-1}$.

GC-MS raw data were processed by an untargeted metabolomics approach as previously described by Cordovez et al. (2015). VOCs were identified by comparison of the mass spectra with those of authentic reference standards and by spectra of NIST08 (National Institute of Standards and Technology, United States), Wiley libraries and the Wageningen Mass Spectral Database of Natural Products and by comparing the experimentally calculated LRI with the literature values. LRI were calculated based on a series of alkanes using a third order polynomial fitting. Three replicates per treatment were used and vials containing $1 / 5^{\text {th }} \mathrm{PDA}$ medium only were used as controls.

\section{Plant Exposure to Synthetic Volatiles}

To investigate if the fungal compounds detected by GC-MS analysis were responsible for the plant growth-promoting effects, we used single and a mix of synthetic compounds for which authentic reference standards were available. The synthetic compounds 2-methyl-1-propanol (analytical standard), 2-pentanone (99.5\%), 2-methyl-1-butanol ( $\geq 98.0 \%)$, 1-octen-3-ol (98\%) and 3-octanone ( $\geq 98 \%)$ were purchased from Sigma-Aldrich. Experiments were performed in Petri dishes ( $\varnothing 90 \mathrm{~mm}$ ) with two-compartments. Four 7-dayold $A$. thaliana seedlings were placed on one compartment containing $0.5 x M S$ medium and different dilutions of the five synthetic compounds and the mixture were applied to a sterile filter paper $(1.5 \mathrm{~cm} \times 1.5 \mathrm{~cm})$ placed in the other compartment. 

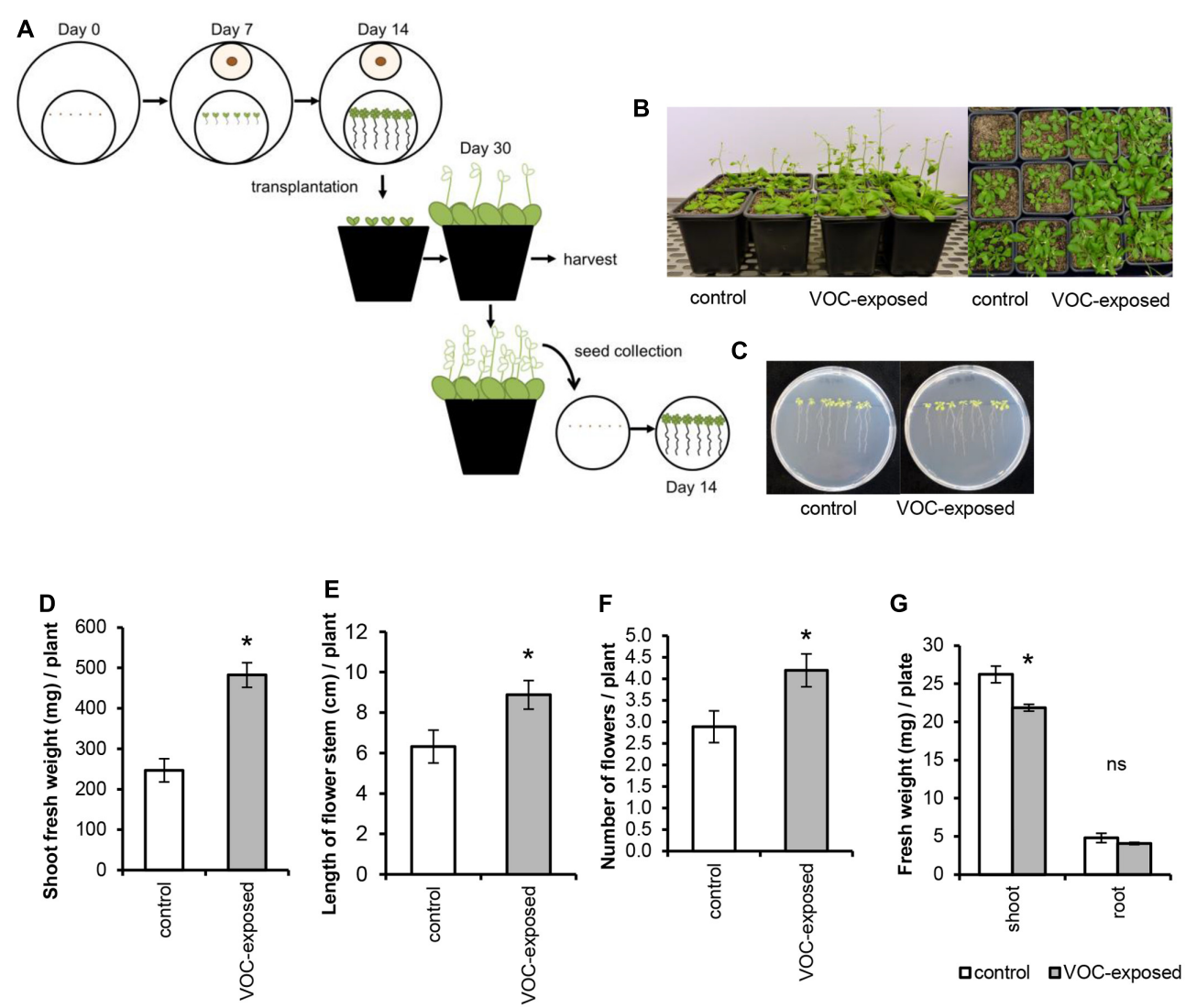

FIGURE 2 | Effects of short exposure of $A$. thaliana to volatile organic compounds (VOCs) produced by R. solani on plant growth and development. Plants exposed to fungal VOCs are referred as 'VOC-exposed' whereas plants exposed to agar medium are referred as 'control.' Asterisks indicate statistically significant differences based on pairwise comparisons between VOC-exposed and control plants (Student's $t$-test, $P<0.05$ ). Non-significant differences are displayed as 'ns'. (A) Schematic representation of the experimental design. (B) Representative pictures of $A$. thaliana plants exposed for 1 week to fungal VOCs or not (control) that were transplanted into a soil-sand mixture and grown for 2 weeks in absence of the VOC-producing fungus. (C) Pictures of 14-day-old seedlings grown from seeds harvested from VOC-exposed plants. (D) Shoot biomass, (E) length of flower stem, and (F) number of flowers (mean \pm SE, $n=12$ ) of VOC-exposed and control plants. (G) Shoot and root biomass (mean \pm SE, $n=4$ ) of seedlings grown from seeds harvested from VOC-exposed plants. Each replicate consists of one Petri dish with eight seedlings.

Dilutions of the synthetic compounds were made as previously described by Blom et al. (2011). Briefly, synthetic compounds were diluted with dichloromethane (DCM) to concentrations of $1 \mathrm{ng}, 10 \mathrm{ng}, 100 \mathrm{ng}, 10 \mu \mathrm{g}, 100 \mu \mathrm{g}$ and $1 \mathrm{mg}$ per $10 \mu \mathrm{L}$. Each compound was mixed in a 1:1 ratio with the lanolin solution (1.6 g of lanolin in $10 \mathrm{~mL} \mathrm{DCM}$ ) and $20 \mu \mathrm{L}$ of this mixture was added to the filter paper. For negative controls, the second compartment was left empty or a mixture of DCM and lanolin was added. For the positive control, seedlings were exposed to the 1-week-old R. solani culture grown on a Petri dish (Ø $35 \mathrm{~mm})$ containing $1 / 5^{\text {th }}$ PDA medium. Petri dishes were immediately sealed and incubated in a growth cabinet. Shoot and root biomass was determined after 2 weeks. Experiments were performed using five biological replicates and statistical differences were determined by pairwise comparison between VOC-exposed and control (exposed to solvent) plants with Student $t$-test (for independent samples) using IBM SPSS Statistics 23. Data were tested for equal variance using Levene's test and for normality using Shapiro-Wilk test at the 5\% significance level.

\section{Plant RNA Extraction, Sequencing and Transcriptome Analyses}

To investigate how VOCs from a soil-borne pathogenic fungus modulate plant growth and development at the transcriptomic level, we sequenced and analyzed the genomewide transcriptome of $A$. thaliana seedlings exposed to $R$. solani VOCs for 7 days in vitro. Seedlings exposed to $1 / 5^{\text {th }}$ PDA medium only were used as control. For the sequencing of plant RNA, total RNA was extracted from roots and shoots. Four replicates were used, and each replicate consisted of four plates with six seedlings each in order to obtain enough biomass. RNA was obtained from frozen tissues with Trizol reagent (Invitrogen). The RNA samples were further purified using the NucleoSpin RNA II kit (Macherey-Nagel). Samples were processed using the NEBNext Ultra Directional RNA Library Prep Kit for Illumina at ServiceXS (GenomeScan B.V., Leiden, The Netherlands). Briefly, mRNA was isolated from the total RNA suing the oligo-dT magnetic beads. After fragmentation of the mRNA, cDNA was synthesized, ligated with sequencing 
adapters and amplified by PCR in order to obtain cDNA libraries. Each cDNA library was individually analyzed for quality and yield using a Fragment Analyzer. cDNA was then clustered and a concentration of $1.6 \mathrm{pM}$ was sequenced with an Illumina NextSeq 500 sequencer. Raw data were submitted to the National Center for Biotechnology Information Short Read Archive (SRA BioProject ID: PRJNA392864, SRA sequences: SAMN07313363 to SAMN07313378).

For the transcriptome analysis, Illumina sequences were trimmed and filtered with FASTQC with a threshold of 25 $(Q>25)$. Quality-trimmed reads were counted using RSEM software package (Li and Dewey, 2011) transformed into RPKM (Reads Per Kilobases per Million reads). Reads were mapped to the A. thaliana reference genes using the software Bowtie2 v.2.1.0 (Langmead and Salzberg, 2012). The Bioconductor package DESeq2 for R Statistical Analysis (Love et al., 2014) was used for normalization and differential expression analyses. The $P$-value was obtained from the differential gene expression test. FDR (False Discovery Rate) correction was used to determine the $P$-value threshold in multiple tests and analyses. Significant differentially expressed genes (DEGs) were selected using FDR $<0.05$ and the absolute value of the $\log _{2}$ Ratio $\geq 0.585$ (at least $1.5 \times$ higher than the expression level in control) as thresholds.

Biological interpretation of the DEGs was carried out with a GO-term enrichment analysis performed using AgriGO $^{1}$. TAIR AGI IDs of significantly up- and down-regulated genes were subjected to a singular enrichment analysis using Fisher test with FDR (Hochberg) at 0.05. Arabidopsis TAIR 9 database was used as a background. Identification of transcription factors (TFs) was carried out using the $A$. thaliana TF database ${ }^{2}$.

\section{Effects of Fungal Volatiles on Plant Resistance to the Fungal Pathogen and Insect Herbivory}

To test the effect of a short exposure to fungal VOCs on plant resistance, 1-week-old $A$. thaliana seedlings were exposed to $R$. solani VOCs for 7 days in vitro and transplanted to sterile potting soil. After 5 and 14 days, plants were inoculated with the fungal root pathogen $R$. solani or infested with the generalist insect herbivore $M$. brassicae, respectively. To test plant resistance to the fungal pathogen, VOC-exposed plants were inoculated with a mycelial plug $(\varnothing 5 \mathrm{~mm})$ of $R$. solani in the proximity of the roots 5 days after soil transplantation $(n=12$ pots containing one plant each). Disease incidence was scored after 7, 10, and 14 days post-inoculation (dpi). Leaves were scored as diseased when they exhibited necrotic or chlorotic lesions. Disease incidence was assessed by determining the percentage of diseased leaves per plant (9-12 plants per treatment). Two independent bioassays were performed. A Generalized Linear Model (GLM, Type III Chi-square Wald test) was adopted to statistically assess the effects of VOC-exposure, time point and their interactions on disease incidence. VOC exposure and time point were used

${ }^{1}$ http://bioinfo.cau.edu.cn/agriGO/index.php

${ }^{2}$ http://arabidopsis.med.ohio-state.edu/ as factors. Main effects of VOC exposure and time point as well as the interactions of VOC exposure $\times$ time point were analyzed. In addition, the main effects of the interaction of VOC exposure, total number of leaves and time point were tested. Least Significant Difference (LSD) test was used to adjust for multiple comparisons. Statistical analyses were performed using IBM SPSS Statistics 23.

To test plant resistance to insects, VOC-exposed plants were infested with 30 neonates of $M$. brassicae using a fine brush ( $n=8$ pots containing three plants each) 2 weeks after soil transplantation. Plant pots were kept inside a plastic container (Duchefa, Haarlem, The Netherlands; height: $140 \mathrm{~mm}$, upper $\varnothing: 115 \mathrm{~mm}$, lower $\varnothing: 90 \mathrm{~mm}$ ), covered with insect-proof mesh cloth and sealed with elastic bands and kept in growth cabinet. Caterpillar density was reduced to 15 and 10 larvae at 3 and 7 days post-infestation (dpi), respectively. The reduction of the herbivore number was used to simulate dispersion and predation in nature. Larval performance was measured by weighing the larvae on a microbalance (accuracy $= \pm 1 \mu \mathrm{g}$; Mettler-Toledo MT5 Electrobalance) at 3, 7, and 10 dpi. A Generalized Linear Model (GLM, Type III Chi-square Wald test) was performed to statistically assess the effects of VOC-exposure, time, replicate and their interactions on larval weight. VOC exposure, time point and replicate were used as factors. Main effects of VOC exposure, time point and replicate as well as the interactions of VOC exposure $x$ time point and VOC exposure $\times$ replicate were analyzed. Bonferroni test was used to adjust for multiple comparisons. Statistical analyses were performed using IBM SPSS Statistics 23.

\section{RESULTS}

\section{Volatiles from the Fungal Pathogen Promote Plant Growth and Prime Plant Development}

To study VOC-mediated interactions, A. thaliana seedlings were co-cultivated with, but physically separated from, the fungal pathogen R. solani (Figure 1A). Seedlings exposed to VOCs from $R$. solani (hereafter referred to as 'VOC-exposed') grown on $1 / 5^{\text {th }}$ PDA medium showed an increase in shoot and root dry weight of $150 \%$ (Mann-Whitney $U$ test, $P=0.008$ ) and $265 \%$ (Mann-Whitney $U$ test, $P=0.008$ ), respectively, compared to seedlings not exposed to the fungal VOCs (hereafter referred to as 'control') (Figure 1B). Also seedlings exposed to VOCs from fungal mycelium inoculated into a soil-sand mixture showed a $62 \%$ increase in root dry weight (Figure $1 B$, $t$-test, $P=0.002$ ). We also tested if VOCs emitted from sclerotia, the survival structures of $R$. solani in soil, affect plant growth. Results showed that also sclerotial VOCs increased shoot and root dry weight by $60 \%$ ( $t$-test, $P<0.001$ ) and $44 \%$ ( $t$-test, $P=0.012$ ), respectively (Figure 1B). Since the production of VOCs by microorganisms can be influenced by the substrate composition, we tested, in addition to the $1 / 5^{\text {th }}$ PDA medium, four other growth media with a composition ranging from nutrient-poor to nutrient-rich. For all tested media, except for water agar, dry weights of plant shoots 
and roots were significantly higher in the fungal VOC-exposed seedlings than in the control (see Supplementary Figure S1).

To study if $R$. solani VOCs can modulate A. thaliana development, we investigated their effects on seed germination as well as radicle and cotyledon emergence. Fungal VOCs had no effect on seed germination nor on cotyledon emergence. However, a delay in radicle emergence was observed for VOCexposed seeds (Figure 1C, Mann-Whitney $U$ test, $P=0.008$ ). Seven days after exposure of the seeds to the fungal VOCs, seedlings displayed shorter primary roots compared to the control (Figure 1D; $t$-test, $P<0.001$ ). Nevertheless, significant plant growth-promoting effects were observed after 18 days, albeit to a lower extent than observed for seedlings (instead of seeds) exposed to the fungal VOCs (Figures 1E,F; ANOVA, $P<0.05)$. To further investigate if $R$. solani VOCs can prime development of plants, $A$. thaliana seedlings were pre-exposed in vitro to fungal VOCs for 7 days, transplanted to a soilsand mixture and grown in absence of the fungus (Figure 2A). After 2 weeks, plants pre-exposed to fungal VOCs showed significant increases in shoot weight, in length of the flower stem and in number of flowers of 96,40 , and $45 \%$, respectively (Figures 2B,D-F). For 10 VOC-exposed and 10 control plants, seeds were collected and subsequently grown on $0.5 x M S$ agar medium to determine if the VOC-mediated effects on shoot and root growth were transmitted to the next generation of A. thaliana. Seedlings originating from seeds of VOCs-exposed plants showed no difference in root weight $(t$-test, $P=0.216)$ and even a slight reduction in shoot weight ( $t$-test, $P=0.034)$ compared to seedlings originating from seeds of control plants (Figures 2C,G).

Collectively, these results indicate that short- and longterm exposure of different developmental stages of A. thaliana, including seed imbibition, to the fungal VOCs significantly enhanced shoot and root biomass, altered root architecture and accelerated development and in particular flowering. The results also suggest that the VOC-mediated effects do not seem be transgenerational although more generations need to be tested to further support this finding.

\section{Identification of Fungal Volatiles and Their Effects on Plant Growth}

Previous studies have reported increased $\mathrm{CO}_{2}$ concentrations in closed systems due to microbial catabolism. Higher $\mathrm{CO}_{2}$ concentrations may lead to enhanced photosynthetic efficiency and increased plant biomass (Poorter and Navas, 2003; Kai and Piechulla, 2009; Jauregui et al., 2015). To evaluate a role of $\mathrm{CO}_{2}$ in the observed plant growth-promoting effects by R. solani VOCs, our first approach was to trap $\mathrm{CO}_{2}$ with different amounts of the $\mathrm{CO}_{2}$ absorber, sodalime. The results showed that even small amounts of sodalime were harmful to the growth of $A$. thaliana seedlings, suggesting a deleterious depletion of $\mathrm{CO}_{2}$ (see Supplementary Figure S2A). A second approach involved exposing the seedlings to elevated $\mathrm{CO}_{2}$ concentration of $1330 \mathrm{ppm}$, which is approximately three times higher than the ambient $\mathrm{CO}_{2}$ concentration of $400 \mathrm{ppm}$. After 7 days of plant growth, no significant differences in plant shoot $(t$-test,
$P=0.219)$ and root (Kruskal-Wallis test, $P=0.389$ ) weights were observed between $\mathrm{CO}_{2}$-exposed and control seedlings (see Supplementary Figures S2B,C).

To determine if ethylene, a volatile hormone produced by several microorganisms (Ilag and Curtis, 1968; Considine et al., 1977), was involved in the plant growth-promoting effects, we first determined if $R$. solani produced ethylene under the growth conditions used in the bioassay. Among the VOCs emitted by a 10-day-old fungal culture, no differences in ethylene concentration were found relative to the control (see Supplementary Figure S3). Since we cannot exclude that ethylene production occurs at earlier or later growth stages of $R$. solani or during its interaction with plants, we tested two independent ethylene-insensitive $A$. thaliana mutants for their response to the fungal VOCs. These mutants were etr1-4 (ethylene receptor mutant) and ein 3 eill (TF double mutant). The results showed that VOC-mediated plant growth promotion was observed for both ethylene-insensitive mutants and to the same extent as observed in wild-type A. thaliana Col-0 (Figures 3A,B). In these assays, we also included Verticillium dahliae, a soil-borne pathogenic fungus that produces ethylene when grown on $1 / 5^{\text {th }}$ PDA. V. dahliae still promoted plant growth in the two ethyleneinsensitive A. thaliana mutants (data not shown). Collectively, these results indicate that ethylene does not play a major role in the plant growth promotion by $R$. solani VOCs observed in our experimental system.

Subsequent GC-MS analysis revealed a total of 14 VOCs in the headspace of $R$. solani cultures that were significantly different from the control (agar medium only) $(t$-test, $P<0.05)$ and with peak intensities at least twice as high as those in the control (Table 1). Among these, we detected the typical fungal VOCs such as 1-octen-3-ol and 3-octanone. Five VOCs, for which authentic reference standards were available, were tested for their effects on plant growth. When $A$. thaliana seedlings were exposed to 2-methyl-1-propanol, 2-pentanone, 2-methyl-1-butanol, 1octen-3-ol or 3-octanone at concentrations ranging from $1 \mathrm{ng}$ to $1 \mathrm{mg}$ per $10 \mu \mathrm{l}$ or a mix of all five synthetic volatile compounds, no growth promotion was observed compared to seedlings exposed to the solvent only, whereas VOCs from $R$. solani showed a significant growth promotion (see Supplementary Figure S4A). Higher concentrations (10 $\mu \mathrm{g}, 100 \mu \mathrm{g}$ and $1 \mathrm{mg}$ ) of 1-octen3-ol, 3-octanone and the mix even inhibited plant growth (see Supplementary Figure S4B). These results indicate that other concentrations or other mixtures of the tested VOCs, or other VOCs not detected by the analytical method used here are involved in plant growth promotion by $R$. solani.

\section{Fungal Volatiles Induce Plant Genome-Wide Transcriptional Changes}

To begin to understand the molecular mechanisms underlying plant growth promotion by VOCs from the fungal root pathogen $R$. solani, we performed genome-wide transcriptome analysis. Significant DEGs were selected with a $\log _{2}$ fold change $\geq+0.058$ $(1.5 \times$ higher $)$ or $\leq-0.585(1.5 \times$ lower $)$ and FDR $<0.05$. Following these criteria, a total of 477 (267 up-regulated and 210 down-regulated) and 12 (6 up-regulated and 6 down-regulated) 

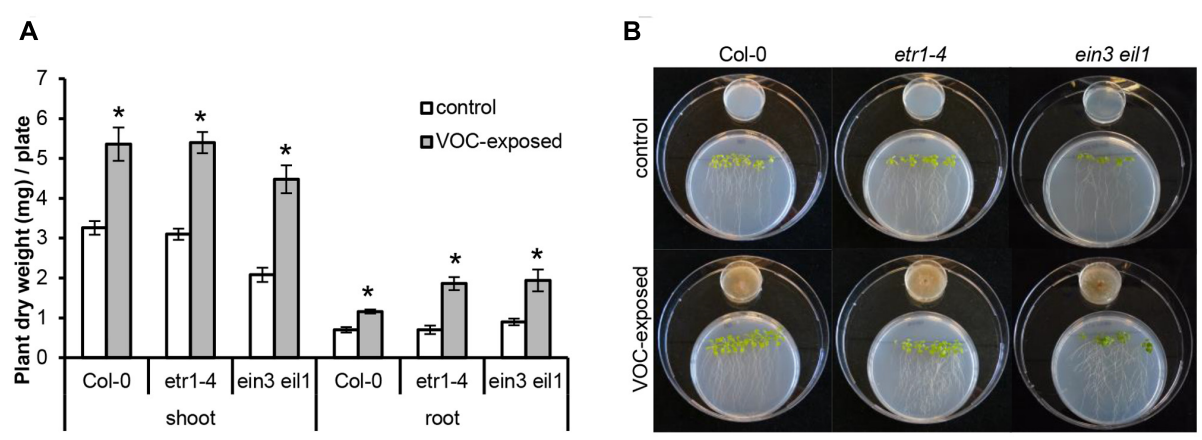

FIGURE 3 | Effects of volatile organic compounds (VOCs) from $R$. solani on the growth of ethylene-insensitive mutants of $A$. thaliana. (A) Shoot and root biomass (mean $\pm \mathrm{SE}, n=4-5$ ) of $A$. thaliana wild-type Col-0 and mutants etr1-4 and ein3 eil1 exposed to VOCs from $R$. solani. Each replicate consists of one Petri dish with six seedlings. Asterisks indicate a statistically significant difference between VOC-exposed and control (exposed to agar medium only) plants (Student's $t$-test, $P<0.05)$. (B) Phenotype of $A$. thaliana wild-type and mutants after 14 days of exposure to the fungal VOCs.

TABLE 1 | Volatile organic compounds (VOCs) emitted by the fungal pathogen Rhizoctonia solani. VOCs displayed are significantly different (Student's $t$-test, $P$ < 0.05 , $n=3$ ), being at least twice as abundant as in the control (medium only). Compounds were identified by comparing their mass spectra and LRI with those of authentic reference standards (MSI level 1) or with spectra and LRI published in the NIST08 and in-house mass spectral libraries (MSI level 2).

\begin{tabular}{|c|c|c|c|c|}
\hline \multirow[b]{2}{*}{ Compound } & \multirow[b]{2}{*}{$\mathbf{R I}^{\mathbf{a}}$} & \multirow[b]{2}{*}{ Annotation $^{\mathbf{b}}$} & \multicolumn{2}{|c|}{ VOC emission } \\
\hline & & & control & R. solani \\
\hline 2-methyl-1-propanol* & 665 & 1 & $258225 \pm 59955$ & $739090 \pm 106560$ \\
\hline Unknown & 706 & 4 & $3664 \pm 1222$ & $318349 \pm 121863$ \\
\hline 2-pentanone* & 709 & 1 & $145709 \pm 18534$ & $329667 \pm 47446$ \\
\hline Methyl thiocyanate & 732 & 2 & $17812 \pm 8687$ & $583890 \pm 36242$ \\
\hline 2-methylbutanenitrile & 740 & 1 & $24682 \pm 18783$ & $414931 \pm 118132$ \\
\hline 3-methyl-butanenitrile & 745 & 1 & $72109 \pm 11955$ & $1169000 \pm 273882$ \\
\hline 2-methyl-1-butanol* & 751 & 1 & $33574 \pm 4041$ & $470300 \pm 181198$ \\
\hline Unknown & 956 & 4 & $11654 \pm 10118$ & $30769 \pm 5295$ \\
\hline 1-octen-3-ol* & 978 & 1 & $4620 \pm 2684$ & $2255670 \pm 1373443$ \\
\hline 3-octanone* & 986 & 1 & $12785 \pm 10514$ & $3549774 \pm 1893459$ \\
\hline 2-ethyl-1-hexanol & 1027 & 2 & $264835 \pm 59710$ & $507060 \pm 88147$ \\
\hline 2-nonen-1-ol & 1172 & 1 & $1956 \pm 281$ & $4839 \pm 1900$ \\
\hline Unknown & 1343 & 4 & $3468 \pm 869$ & $5971 \pm 1257$ \\
\hline Alpha-copaene & 1403 & 2 & $5092 \pm 3553$ & $45013 \pm 21268$ \\
\hline
\end{tabular}

${ }^{\text {a }}$ Calculated retention indices $(R I)$.

${ }^{b}$ Annotation level according to the Metabolomics Standards Initiative (MSI).

${ }^{c}$ Emission values refer to peak intensity (mean $\pm S D$ ).

*Compounds tested for plant-growth promoting effects.

genes were identified in A. thaliana shoot and root tissues exposed to R. solani VOCs, respectively (see Supplementary Table S1). To identify enriched Gene Ontology (GO) terms, a singular enrichment analysis (SEA) of GO categories was conducted using AgriGO (see Supplementary Tables S2, S3). Transcriptional changes in genes involved in growth and resistance of $A$. thaliana are highlighted in Figure 4.

Among the GO terms identified for shoot up-regulated genes, we found an enrichment of genes involved in photosynthesis and auxin response. Since plant hormones have an essential role in integrating plant growth, development and resistance, we zoomed in on the GO-term 'hormone-mediated signaling pathway' to further investigate how fungal VOCs affect the balance of these processes in the A. thaliana plants. Up-regulated shoot genes in this category included genes involved in auxin and abscisic acid mediated (ABA) signaling pathways. Genes involved in auxin homeostasis included IAA-2 (AT3G23030.1), IAA-19 (AT3G15540.1), IAA-29 (AT4G32280.1), WES1 (AT4G27260.1), HAT2 (AT5G47370.1), and DFL1 (AT5G54510.1) (Figure 4). Also, other genes involved in the response to auxin, such as PIF5 (AT3G59060) and HB-2 (AT4G16780.1), were found among the up-regulated genes. In addition, CYP707A3 (AT5G45340.1), involved in ABA catabolic process, was 6.3-fold up-regulated in root genes of VOC-exposed seedlings. Down-regulated shoot genes included genes involved in ethylene and jasmonic acid (JA) signaling pathways. Genes involved in response to gibberellin (GA) and brassinosteroid (BR) stimuli were both up- and down-regulated in shoot tissue (see Supplementary Tables S4, S5). 


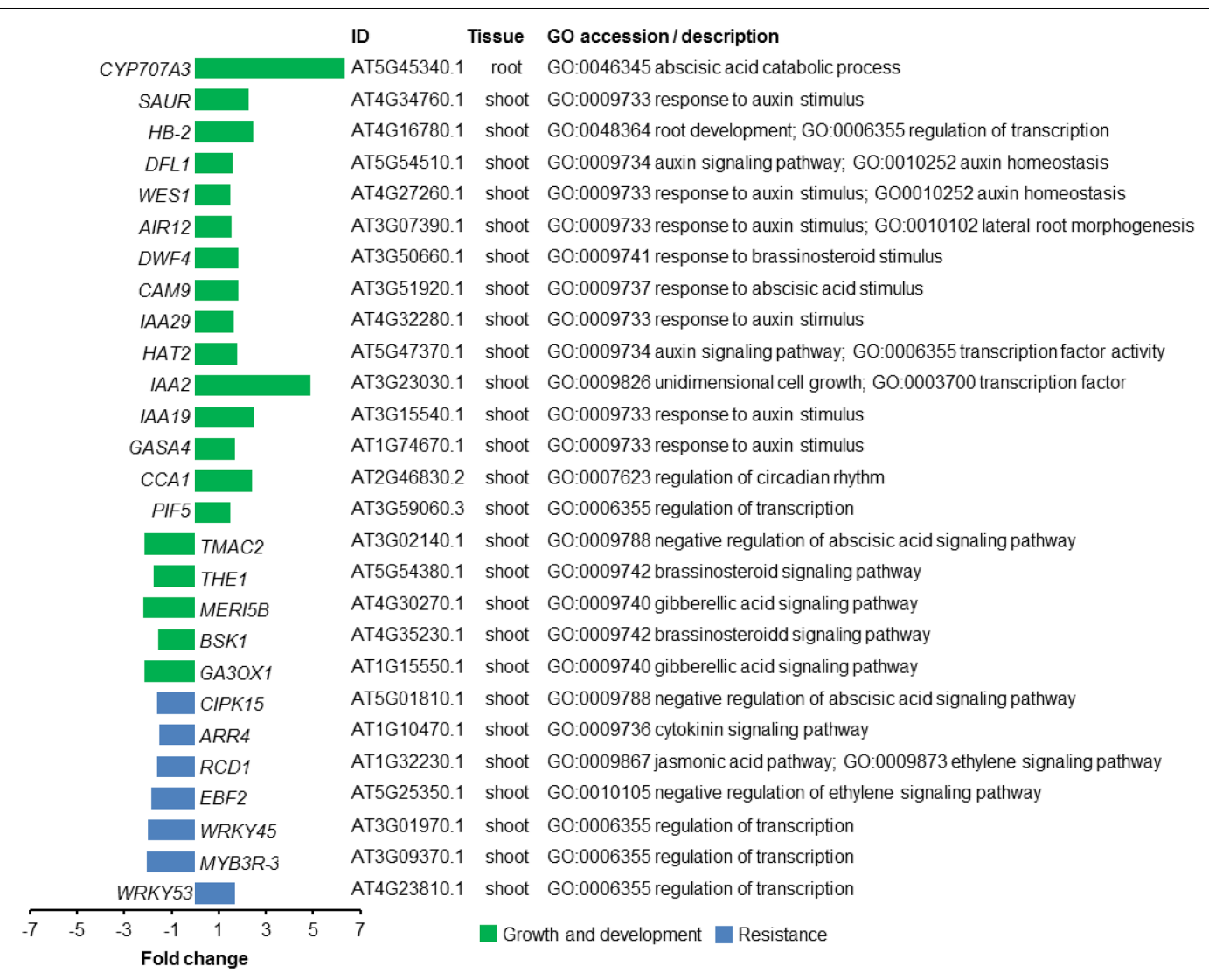

FIGURE 4 | Transcriptional changes in genes of $A$. thaliana exposed to volatile organic compounds (VOCs) from R. solani. Differentially expressed genes (DEGs) for seedlings exposed to the fungal VOCs are shown. Genes described to be involved in plant growth and development are depicted in green, whereas genes associated with resistance responses to biotic stress are depicted in blue. Gene Ontology (GO) description is based on the output from AgriGO.

It was not possible to perform an enrichment analysis for root DEGs since the total number of DEGs was lower than the required minimum number of genes in the query. Given the up-regulation of genes involved in the response to auxin, we tested a number of A. thaliana mutants impaired in auxin perception (tir1 afb1), biosynthesis (wei8), the PHYTOCHROME INTERACTING FACTOR (pif4 pif5) as well as an overexpressor of a stable version of the negative regulator of PIF activity HFR1 (G-BH-03) and the $h f r 1$ knockout mutant. The results showed that VOC-mediated plant growth promotion was observed for all mutants to a similar or greater extent as observed for wild-type A. thaliana Col-0 (see Supplementary Figure S5). These results suggest that the PIF-auxin axis of growth regulation does not play a major role in the growth-promoting effects of $A$. thaliana seedlings exposed to $R$. solani VOCs.

To study changes in the expression of regulatory genes of A. thaliana induced by $R$. solani VOCs, we also identified TFs encoded by the DEGs using a web-based tool ${ }^{3}$. We found a total of 20 and 22 TFs that were up- and downregulated, respectively, when seedlings were exposed to the fungal VOCs (see Supplementary Table S6). Previous studies with $A$. thaliana have demonstrated that members of the

${ }^{3} \mathrm{http}: / /$ arabidopsis.med.ohio-state.edu/AtTFDB
WRKY and the MYB TF families control various processes involved in the responses to biotic and abiotic stresses, development, metabolism, and defense. Our analyses identified several members of the MYB and the WRKY families: WRKY53 (AT4G23810) was up-regulated, whereas WRKY26 (AT5G07100), WRKY39 (AT3G04670), WRKY45 (AT3G01970), and MYB3R-3 (AT3G09370) were down-regulated in plants exposed to fungal VOCs. Some of these down-regulated TFs have been described for their role in plant resistance to abiotic stresses, such as WRKY26 and WRKY39, and to biotic stresses, such as WRKY45 (Li et al., 2011; Shimono et al., 2012; Cheng et al., 2015).

\section{Fungal Volatiles Affect Plant Resistance to Insect Herbivores But Not to the VOC-Producing Fungal Pathogen}

To determine if VOCs can modulate disease resistance, we investigated if plants that were previously exposed to the VOCs emitted by $R$. solani had become more resistant to infection by $R$. solani itself (Figure $\mathbf{5 A}$ ). The overall results of two independent bioassays showed that $R$. solani disease incidence of VOC-exposed plants, monitored at different time points, did not differ from that of the control plants (Figure 5B; GLM, $P=0.836$ ). In the second bioassay, disease incidence at $7 \mathrm{dpi}$ 

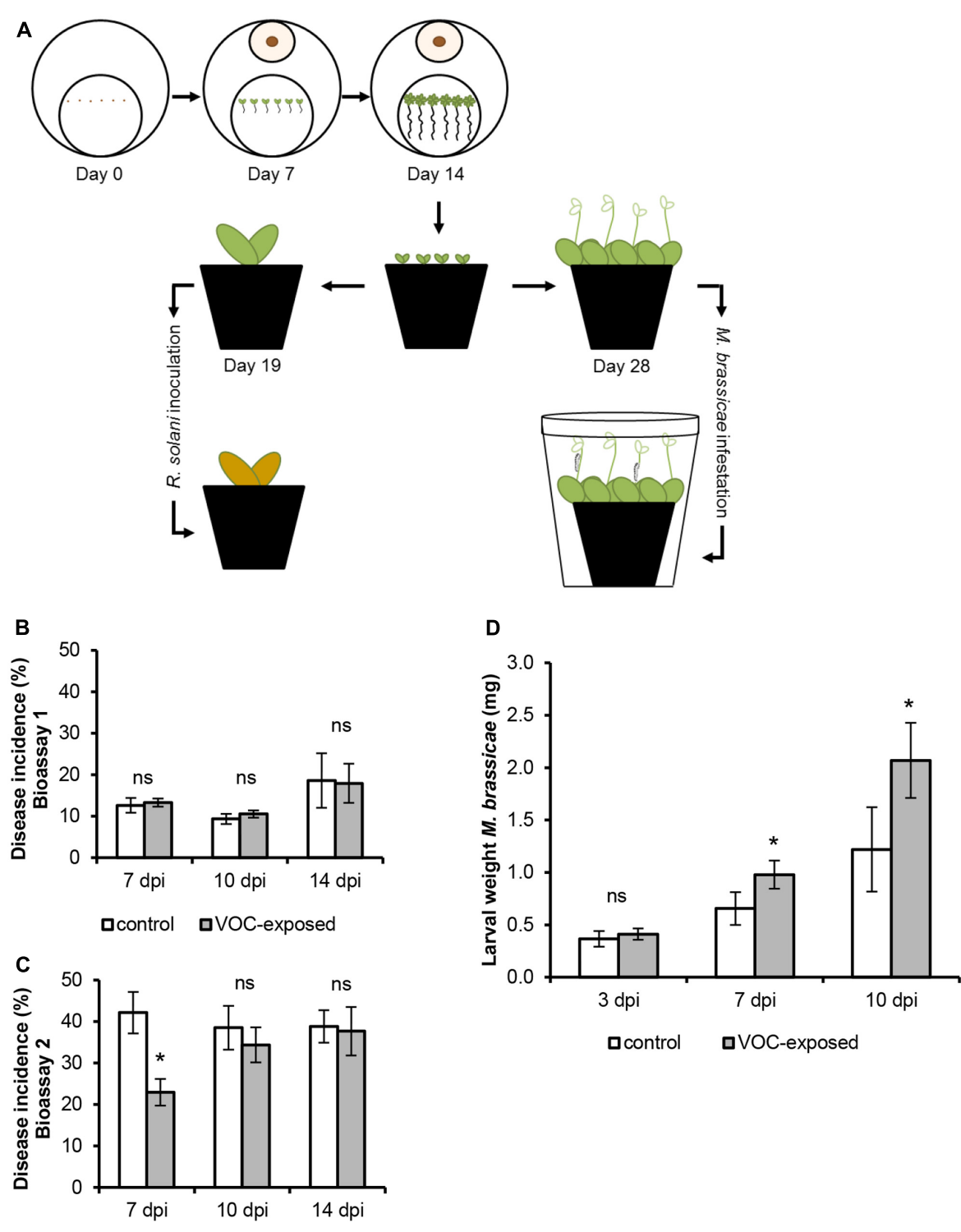

FIGURE 5 | Effects of volatile organic compounds (VOCs) from $R$. solani on resistance of $A$. thaliana to fungal infection and insect herbivory. (A) Schematic representation of the experimental set-up to test plant resistance to the fungal pathogen and the insect herbivore. (B,C) Disease incidence (mean \pm SE, $n=9-12)$ of VOC-exposed $A$. thaliana challenged with $R$. solani obtained in two independent bioassays. Disease symptoms were determined at 7 , 10 , and 14 days post inoculation (dpi). (D) Biomass of Mamestra brassicae larvae (mean \pm SE, $n=8$ ) feeding on $A$. thaliana previously exposed to fungal VOCs or to the agar medium only (control). Each pot contained three plants which were infested with 30 neonates. Larval weight was measured at 3 , 7 , and 10 days post-infestation (dpi). Asterisks indicate statistically significant differences based on pairwise comparisons between VOC-exposed and control plants (GLM, $P<0.05)$. Non-significant differences are displayed as 'ns.'

was significantly lower in VOC-exposed plants than in control plants leading to a significant overall effect of the VOC exposure on disease incidence (Figure 5C; GLM, $P=0.025$ ). It should be emphasized, however, that the absolute number of leaves with disease symptoms was similar for control and VOC-exposed plants, however, the increased number of leaves in VOC-exposed plants (see Supplementary Figure S6) resulted in a lower disease incidence. No significant interaction effects were found between the VOC exposure, the total number of leaves and the time points $\left(\mathrm{GLM}, P_{\text {bioassay_1 }}=0.101, P_{\text {bioassay_2 }}=0.139\right)$.
To further investigate the effect of fungal VOCs on aboveground resistance, we exposed plants to the insect herbivore M. brassicae (Figure 5A). Biomass of larvae feeding on leaves of fungal VOC-exposed plants increased in fresh weight by 49 and $70 \%$ at 7 and $10 \mathrm{dpi}$, respectively, as compared to that of larvae feeding on leaves of non-exposed control plants (Figure 5D). Statistically significant effects of the time point (GLM, $P \leq 0.001$ ), VOC exposure (GLM, $P \leq 0.001$ ), replicate (GLM, $P=0.005$ ) as well as the interactions between VOC exposure and time point (GLM, $P \leq 0.001)$ and between VOC 
exposure and replicate (GLM, $P=0.001$ ) were observed. These analyses indicate that exposure of $A$. thaliana to $R$. solani VOCs did not affect belowground resistance to the VOC-producing fungus itself but reduced aboveground resistance to insect larval feeding.

\section{DISCUSSION}

Soil microorganisms can affect plant growth and disease resistance from a distance via the production of VOCs, however, the natural functions, the chemistry and underlying molecular mechanisms are largely unknown. Here, we showed that VOCs from the fungal root pathogen $R$. solani led to an increase in plant biomass, an effect that confirms and extends recent results obtained for plant pathogenic Fusarium and Alternaria species (Bitas et al., 2015; Sanchez-Lopez et al., 2016). No plant growth promotion was observed for five $R$. solani VOCs tested individually and as mixtures, suggesting that other mixtures of the tested VOCs or other VOCs not detected are involved in plant growth promotion by $R$. solani.

The results of this study showed, for the first time, that R. solani VOCs primed and accelerated the development of A. thaliana seedlings leading to a higher number of flowers in VOC-exposed plants. These findings suggest that plants sensing the presence of the fungal root pathogen via VOCs accelerate their development to produce offspring faster. Hence, we hypothesized that VOCs emitted by the pathogenic fungus may function as an 'alert' signal to plants, accelerating growth and development as well as triggering plant resistance in case of a physical encounter with the pathogen. Alternatively, plants may use enhanced growth upon perception of a potential pathogen as a defensive strategy, sacrificing part of the root or shoot biomass and reallocating resources into reproduction rather than in defense processes. Our results showed that exposure of $A$. thaliana to the VOCs from $R$. solani did alter resistance to herbivory aboveground but did not affect resistance belowground to infections by the VOC-producing fungal pathogen itself. Based on these results, we postulate that the fungal pathogen may use VOCs to manipulate, from a distance, plant growth and metabolism to its own benefit. The VOC-induced increase in root biomass and lateral root formation provides a greater root surface area for fungal colonization and infection. Especially for immobile sclerotia, these phenotypic changes in root biomass and architecture would increase the chances of a physical encounter with the roots of host plants.

Previous studies have shown that fungal metabolites can manipulate its host plants to favor invasion and nutrient uptake as well as to counteract the establishment of host immunity (Biemelt and Sonnewald, 2006; Chanclud and Morel, 2016). For example, Tuber species have been shown to manipulate ethylene pathways in its host by the production of this hormone, inducing root morphological modifications (Splivallo et al., 2009). In our study, no evidence was found for a role of ethylene in the phenotypic responses induced in A. thaliana by $R$. solani VOCs. In addition to hormones, oxylipins, have been proposed as developmental and communication signals between fungi and plants (Tsitsigiannis and Keller, 2007). In plants, oxylipins are involved in the regulation of plant growth and development as well as defense to biotic and abiotic stresses, whereas in fungi they are used as a developmental signal for fungal spore germination and growth (Brodhun and Feussner, 2011). Several lines of evidence suggest that plant oxylipins can be partly substituted for fungal oxylipins and that fungal oxylipins can influence plant development by mimicking the plant endogenous signal communication (Tsitsigiannis and Keller, 2007; Brodhagen et al., 2008). VOC profiling of $R$. solani showed the presence of the oxylipins 1-octen-3-ol and 3-octanone, but these and other VOCs did not show a significant effect on plant growth when tested individually or in a mixture; several were even toxic at higher concentrations.

To begin to understand the molecular mechanisms underlying VOC-mediated interactions between A. thaliana and R. solani, we looked into the transcriptional changes triggered by plant exposure to the fungal VOCs. Our genome-wide transcriptome analysis revealed that $R$. solani VOCs modulated the expression of genes involved in auxin and ABA responses as well as photosynthesis. Among the up-regulated genes, we observed an enrichment of the genes involved in auxin homeostasis such as IAA2, IAA19, IAA29, HAT2, DFL1, and WES1. Auxin is a common target for the manipulation of plant development and resistance by either beneficial or pathogenic microorganisms (Grunewald et al., 2009; Cui et al., 2013). This hormone stimulates plant growth which can then be exploited by these microorganisms. In addition, auxin can also favor pathogen infection, for example, by increasing expansin production which in turn facilitates penetration of the plant cell wall (Pusztahelyi et al., 2015). In a recent study, Garnica-Vergara et al. (2016) showed that auxin signaling in A. thaliana is affected by the volatile 6-pentyl-2H-pyran-2-one emitted by the beneficial fungus T. atroviride. Also Bitas et al. (2015) showed that F. oxysporum can manipulate auxin transport and signaling via the production of VOCs. Although the results from our transcriptome analyses also pointed to modulation of auxin in seedlings exposed to the VOCs from R. solani, several A. thaliana mutants in auxin perception, biosynthesis and signaling still showed an increase in plant growth when exposed to the fungal VOCs. Thus, it is likely that the mechanisms underlying the plant growth-promoting effect by $R$. solani are mediated via other signaling pathways not detected in our transcriptome analysis. Sanchez-Lopez et al. (2016) have shown that VOCs emitted by A. alternata changed the expression of light- and CK-responsive genes involved in photosynthesis, growth and flowering. The authors concluded that the fungal VOCs promoted plant growth and flowering through cytokinin signaling. Although $R$. solani VOCs also altered genes involved in photosynthesis, we were not able to detect an enrichment of genes involved in cytokinin signaling. Extensive temporal transcriptome analyses of plants exposed to fungal VOCs will be needed to get more detailed insight into the critical time points in the trajectory of plant development, where the fungal VOCs prime essential transcriptional changes that lead to plant growth promotion. 
Interestingly, several of the genes found to be up-regulated in VOC-exposed plants, in particular IAA2, IAA19, IAA29, and HAT2, have been reported to be associated with shadeavoidance responses (de Wit et al., 2014). When competing for light, $A$. thaliana plants invest in shade-avoidance responses such as increased leaf angles, stem elongation, apical dominance and early flowering, phenotypes that allow competition with neighboring plants (Casal, 2013; Ballaré and Pierik, 2017). Our results further show the up-regulation in shoot tissues of PIF5, a phytochrome interacting-factor also involved in shade-avoidance responses. PIF5 regulates elongation growth by controlling the expression of genes that code for auxin biosynthesis and auxin signaling components (Lorrain et al., 2008; Hornitschek et al., 2012). Plant investment in growth during shade-avoidance responses compromises pathogen resistance by repression of JA-dependent defense mechanisms (de Wit et al., 2013). Although VOC exposure showed no change in belowground plant resistance against the fungal pathogen $R$. solani, it did show a negative effect on aboveground resistance to the insect herbivore M. brassicae.

\section{CONCLUSION}

Plant growth promotion by microbial VOCs appears to be a widespread phenomenon, not only observed for beneficial microorganisms but also for fungal root pathogens. Our results indicate that VOCs from a soil-borne pathogenic fungus can prime plant growth and development thereby influencing the trade-off between plant growth/development and resistance to insect herbivores. VOC-mediated interactions are dependent on the dynamics of the whole ecosystem including soil properties and the complex and competitive interactions among (micro)organisms. How the VOC-mediated phenotypic changes in plant growth affect plant chemistry and tri-trophic interactions is yet unknown. Future investigations will further expand on

\section{REFERENCES}

Alonso, J. M., Stepanova, A. N., Solano, R., Wisman, E., Ferrari, S., Ausubel, F. M., et al. (2003). Five components of the ethylene-response pathway identified in a screen for weak ethylene-insensitive mutants in Arabidopsis. Proc. Natl. Acad. Sci. U.S.A. 100, 2992-2997. doi: 10.1073/pnas.0438070100

Ballaré, C. L., and Pierik, R. (2017). The shade-avoidance syndrome: multiple signals and ecological consequences. Plant Cell Environ. doi: 10.1111/pce.12914 [Epub ahead of print].

Bailly, A., and Weisskopf, L. (2012). The modulating effect of bacterial volatiles on plant growth: current knowledge and future challenges. Plant Signal. Behav. 7, 79-85. doi: 10.4161/psb.7.1.18418

Biemelt, S., and Sonnewald, U. (2006). Plant-microbe interactions to probe regulation of plant carbon metabolism. J. Plant Physiol. 163, 307-318. doi: 10.1016/j.jplph.2005.10.011

Bitas, V., Kim, H. S., Bennett, J. W., and Kang, S. (2013). Sniffing on microbes: diverse roles of microbial volatile organic compounds in plant health. Mol. Plant Microbe Interact. 26, 835-843. doi: 10.1094/MPMI-10-12-0249-CR

Bitas, V., McCartney, N., Li, N., Demers, J., Kim, J. E., Kim, H. S., et al. (2015). Fusarium oxysporum volatiles enhance plant growth via affecting auxin transport and signaling. Front. Microbiol. 6:1248. doi: 10.3389/fmicb.2015. 01248 this line of research and focus on effects of microbial VOCs, both from saprophytic and pathogenic soil-borne fungi in their natural habitat. These studies will further contribute to a better understanding of the natural roles of microbial VOCs in the plant root-soil interface.

\section{AUTHOR CONTRIBUTIONS}

VC designed and performed the experiments, carried out the data analyses and drafted the manuscript. LM assisted with the experimental design, $\mathrm{CO}_{2}$ measurements and $\mathrm{CO}_{2}$ exposure assays. KM and DL-B assisted with the insect resistance assay and data analysis. $\mathrm{RP}$ carried out the fungal ethylene measurements, assisted with $\mathrm{CO}_{2}$ exposure assays and provided $A$. thaliana mutants. RM assisted with the fungal VOC collection and analysis. VJC assisted with experimental design and transcriptome analyses. JR coordinated the study and assisted with the experimental design and with drafting the manuscript. All authors agreed with the final version of the manuscript.

\section{ACKNOWLEDGMENTS}

We thank Victor de Jager for the quality check of the transcriptome data and Prof. Dr. Marcel Dicke for the fruitful discussions about this research project. This manuscript is publication number 6327 of Netherlands Institute of Ecology (NIOO-KNAW).

\section{SUPPLEMENTARY MATERIAL}

The Supplementary Material for this article can be found online at: http://journal.frontiersin.org/article/10.3389/fpls.2017.01262/ full\#supplementary-material

Blom, D., Fabbri, C., Connor, E. C., Schiestl, F. P., Klauser, D. R., Boller, T., et al. (2011). Production of plant growth modulating volatiles is widespread among rhizosphere bacteria and strongly depends on culture conditions. Environ. Microbiol. 13, 3047-3058. doi: 10.1111/j.1462-2920.2011.02582.x

Brodhagen, M., Tsitsigiannis, D. I., Hornung, E., Goebel, C., Feussner, I., and Keller, N. P. (2008). Reciprocal oxylipin-mediated cross-talk in the Aspergillusseed pathosystem. Mol. Microbiol. 67, 378-391. doi: 10.1111/j.1365-2958.2007. 06045.x

Brodhun, F., and Feussner, I. (2011). Oxylipins in fungi. FEBS J. 278, 1047-1063. doi: $10.1111 /$ j.1742-4658.2011.08027.x

Casal, J. J. (2013). Photoreceptor signaling networks in plant responses to shade. Annu. Rev. Plant Biol. 64, 403-427. doi: 10.1146/annurev-arplant-050312120221

Chanclud, E., and Morel, J. B. (2016). Plant hormones: a fungal point of view. Mol. Plant Pathol. 17, 1289-1297. doi: 10.1111/mpp.12393

Chang, C., Kwok, S. F., Bleecker, A. B., and Meyerowitz, E. M. (1993). Arabidopsis ethylene-response gene ETR1: similarity of product to twocomponent regulators. Science 262, 539-544. doi: 10.1126/science.8211181

Cheng, H., Liu, H., Deng, Y., Xiao, J., Li, X., and Wang, S. (2015). The WRKY452 WRKY13 WRKY42 transcriptional regulatory cascade is required for rice resistance to fungal pathogen. Plant Physiol. 167, 1087-1099. doi: 10.1104/pp. 114.256016 
Chitarra, G. S., Abee, T., Rombouts, F. M., Posthumus, M. A., and Dijksterhuis, J. (2004). Germination of Penicillium paneum conidia is regulated by 1-octen-3-ol, a volatile self-inhibitor. Appl. Environ. Microbiol. 70, 2823-2829. doi: 10.1128/aem.70.5.2823-2829.2004

Considine, P. J., Flynn, N., and Patching, J. W. (1977). Ethylene production by soil microorganisms. Appl. Environ. Microbiol. 33, 977-979.

Cordovez, V., Carrion, V. J., Etalo, D. W., Mumm, R., Zhu, H., Van Wezel, G. P., et al. (2015). Diversity and functions of volatile organic compounds produced by Streptomyces from a disease-suppressive soil. Front. Microbiol. 6:1081. doi: 10.3389/fmicb.2015.01081

Cui, F., Wu, S., Sun, W., Coaker, G., Kunkel, B., He, P., et al. (2013). The Pseudomonas syringae type III effector AvrRpt2 promotes pathogen virulence via stimulating Arabidopsis auxin/indole acetic acid protein turnover. Plant Physiol. 162, 1018-1029. doi: 10.1104/pp.113.219659

D’Alessandro, M., Erb, M., Ton, J., Brandenburg, A., Karlen, D., Zopfi, J., et al. (2014). Volatiles produced by soil-borne endophytic bacteria increase plant pathogen resistance and affect tritrophic interactions. Plant Cell Environ. 37, 813-826. doi: 10.1111/pce.12220

Das, A., Lee, S.-H., Hyun, T. K., Kim, S.-W., and Kim, J.-Y. (2012). Plant volatiles as method of communication. Plant Biotechnol. Rep. 7, 9-26. doi: 10.1007/s11816012-0236- 1

de Wit, M., Lorrain, S., and Fankhauser, C. (2014). Auxin-mediated plant architectural changes in response to shade and high temperature. Physiol. Plant. 151, 13-24. doi: 10.1111/ppl.12099

de Wit, M., Spoel, S. H., Sanchez-Perez, G. F., Gommers, C. M., Pieterse, C. M., Voesenek, L. A., et al. (2013). Perception of low red:far-red ratio compromises both salicylic acid- and jasmonic acid-dependent pathogen defences in Arabidopsis. Plant J. 75, 90-103. doi: 10.1111/tpj.12203

Dicke, M., and Loreto, F. (2010). Induced plant volatiles: from genes to climate change. Trends Plant Sci. 15, 115-117. doi: 10.1016/j.tplants.2010.01.007

Effmert, U., Kalderas, J., Warnke, R., and Piechulla, B. (2012). Volatile mediated interactions between bacteria and fungi in the soil. J. Chem. Ecol. 38, 665-703. doi: 10.1007/s10886-012-0135-5

Ezra, D., and Strobel, G. A. (2003). Effect of substrate on the bioactivity of volatile antimicrobials produced by Muscodor albus. Plant Sci. 165, 1229-1238. doi: 10.1016/s0168-9452(03)00330-3

Galstyan, A., Cifuentes-Esquivel, N., Bou-Torrent, J., and Martinez-Garcia, J. F. (2011). The shade avoidance syndrome in Arabidopsis: a fundamental role for atypical basic helix-loop-helix proteins as transcriptional cofactors. Plant J. 66, 258-267. doi: 10.1111/j.1365-313X.2011.04485.x

Garnica-Vergara, A., Barrera-Ortiz, S., Munoz-Parra, E., Raya-Gonzalez, J., Mendez-Bravo, A., Macias-Rodriguez, L., et al. (2016). The volatile 6pentyl-2H-pyran-2-one from Trichoderma atroviride regulates Arabidopsis thaliana root morphogenesis via auxin signaling and ETHYLENE INSENSITIVE 2 functioning. New Phytol. 209, 1496-1512. doi: 10.1111/nph. 13725

Grunewald, W., Van Noorden, G., Van Isterdael, G., Beeckman, T., Gheysen, G., and Mathesius, U. (2009). Manipulation of auxin transport in plant roots during Rhizobium symbiosis and nematode parasitism. Plant Cell 21, 2553-2562. doi: 10.1105/tpc.109.069617

Herrero-Garcia, E., Garzia, A., Cordobes, S., Espeso, E. A., and Ugalde, U. (2011). 8-Carbon oxylipins inhibit germination and growth, and stimulate aerial conidiation in Aspergillus nidulans. Fungal Biol. 115, 393-400. doi: 10.1016/j. funbio.2011.02.005

Hornitschek, P., Kohnen, M. V., Lorrain, S., Rougemont, J., Ljung, K., LopezVidriero, I., et al. (2012). Phytochrome interacting factors 4 and 5 control seedling growth in changing light conditions by directly controlling auxin signaling. Plant J. 71, 699-711. doi: 10.1111/j.1365-313X.2012. 05033.x

Hung, R., Lee, S., and Bennett, J. W. (2013). Arabidopsis thaliana as a model system for testing the effect of Trichoderma volatile organic compounds. Fungal Ecol. 6, 19-26. doi: 10.1016/j.funeco.2012.09.005

Ilag, L., and Curtis, R. W. (1968). Production of ethylene by fungi. Science 159, 1357-1358. doi: 10.1126/science.159.3821.1357

Jauregui, I., Aparicio-Tejo, P. M., Avila, C., Rueda-Lopez, M., and Aranjuelo, I. (2015). Root and shoot performance of Arabidopsis thaliana exposed to elevated $\mathrm{CO}_{2}$ : a physiologic, metabolic and transcriptomic response. J. Plant. Physiol. 189, 65-76. doi: 10.1016/j.jplph.2015.09.012
Junker, R. R., and Tholl, D. (2013). Volatile organic compound mediated interactions at the plant-microbe interface. J. Chem. Ecol. 39, 810-825. doi: 10.1007/s10886-013-0325-9

Kai, M., and Piechulla, B. (2009). Plant growth promotion due to rhizobacterial volatiles - an effect of $\mathrm{CO}_{2}$ ? FEBS Lett. 583, 3473-3477. doi: 10.1016/j.febslet. 2009.09.053

Kegge, W., and Pierik, R. (2010). Biogenic volatile organic compounds and plant competition. Trends Plant Sci. 15, 126-132. doi: 10.1016/j.tplants.2009.11.007

Kottb, M., Gigolashvili, T., Grosskinsky, D. K., and Piechulla, B. (2015). Trichoderma volatiles effecting Arabidopsis: from inhibition to protection against phytopathogenic fungi. Front. Microbiol. 6:995. doi: 10.3389/fmicb. 2015.00995

Langmead, B., and Salzberg, S. L. (2012). Fast gapped-read alignment with Bowtie 2. Nat. Methods 9, 357-359. doi: 10.1038/nmeth.1923

Lee, B., Farag, M. A., Park, H. B., Kloepper, J. W., Lee, S. H., and Ryu, C. M. (2012). Induced resistance by a long-chain bacterial volatile: elicitation of plant systemic defense by a C13 volatile produced by Paenibacillus polymyxa. PLoS ONE 7:e48744. doi: 10.1371/journal.pone.0048744

Li, B., and Dewey, C. N. (2011). RSEM: accurate transcript quantification from RNA-Seq data with or without a reference genome. BMC Bioinformatics 12:323. doi: 10.1186/1471-2105-12-323

Li, S., Fu, Q., Chen, L., Huang, W., and Yu, D. (2011). Arabidopsis thaliana WRKY25, WRKY26, and WRKY33 coordinate induction of plant thermotolerance. Planta 233, 1237-1252. doi: 10.1007/s00425-011-1375-2

Lorrain, S., Allen, T., Duek, P. D., Whitelam, G. C., and Fankhauser, C. (2008). Phytochrome-mediated inhibition of shade avoidance involves degradation of growth-promoting bHLH transcription factors. Plant J. 53, 312-323. doi: 10. 1111/j.1365-313X.2007.03341.x

Love, M. I., Huber, W., and Anders, S. (2014). Moderated estimation of fold change and dispersion for RNA-seq data with DESeq2. Genome Biol. 15:550. doi: 10.1186/s13059-014-0550-8

Morath, S. U., Hung, R., and Bennett, J. W. (2012). Fungal volatile organic compounds: a review with emphasis on their biotechnological potential. Fungal Biol. Rev. 26, 73-83. doi: 10.1016/j.fbr.2012.07.001

Mumm, R., and Dicke, M. (2010). Variation in natural plant products and the attraction of bodyguards involved in indirect plant defense. Can. J. Zool. 88, 628-667. doi: 10.1139/z10-032

Murashige, T., and Skoog, F. (1962). A revised medium for rapid growth and bioassays with tobacco tissue cultures. Physiol. Plant. 15, 473-497.

Paré, P. W., and Tumlinson, J. H. (1999). Plant volatiles as a defense against insect herbivores. Plant Physiol. 121, 325-331.

Park, Y. S., Dutta, S., Ann, M., Raaijmakers, J. M., and Park, K. (2015). Promotion of plant growth by Pseudomonas fluorescens strain SS101 via novel volatile organic compounds. Biochem. Biophys. Res. Commun. 461, 361-365. doi: 10.1016/j.bbrc. 2015.04.039

Pierik, R., Djakovic-Petrovic, T., Keuskamp, D. H., De Wit, M., and Voesenek, L. A. (2009). Auxin and ethylene regulate elongation responses to neighbor proximity signals independent of gibberellin and della proteins in Arabidopsis. Plant Physiol. 149, 1701-1712. doi: 10.1104/pp.108.133496

Poorter, H., and Navas, L. M. (2003). Plant growth and competition at elevated $\mathrm{CO}_{2}$ : on winners, losers and functional groups. New Phytol. 157, 175-198. doi: 10.1046/j.1469-8137.2003.00680.x

Pusztahelyi, T., Holb, I. J., and Pócsi, I. (2015). Secondary metabolites in fungusplant interactions. Front. Plant Sci. 6:573. doi: 10.3389/fpls.2015.00573

Roze, L. V., Beaudry, R. M., and Linz, J. E. (2012). Analysis of volatile compounds emitted by filamentous fungi using solid-phase microextractiongas chromatography/mass spectrometry. Methods Mol. Biol. 944, 133-142. doi: 10.1007/978-1-62703-122-6_9

Ryu, C. M., Farag, M. A., Hu, C. H., Reddy, M. S., Kloepper, J. W., and Pare, P. W. (2004). Bacterial volatiles induce systemic resistance in Arabidopsis. Plant Physiol. 134, 1017-1026. doi: 10.1104/pp.103.026583

Ryu, C. M., Farag, M. A., Hu, C. H., Reddy, M. S., Wei, H. X., Pare, P. W., et al. (2003). Bacterial volatiles promote growth in Arabidopsis. Proc. Natl. Acad. Sci. U.S.A. 100, 4927-4932. doi: 10.1073/pnas.0730845100

Sanchez-Lopez, A. M., Baslam, M., De Diego, N., Munoz, F. J., Bahaji, A., Almagro, G., et al. (2016). Volatile compounds emitted by diverse phytopathogenic microorganisms promote plant growth and flowering through cytokinin action. Plant Cell Environ. 39, 2592-2608. doi: 10.1111/pce.12759 
Schmidt, R., Cordovez, V., De Boer, W., Raaijmakers, J., and Garbeva, P. (2015). Volatile affairs in microbial interactions. ISME J. 9, 2329-2335. doi: 10.1038/ ismej.2015.42

Schulz, S., and Dickschat, J. S. (2007). Bacterial volatiles: the smell of small organisms. Nat. Prod. Rep. 24, 814-842. doi: 10.1039/b507392h

Sessa, G., Carabelli, M., Sassi, M., Ciolfi, A., Possenti, M., Mittempergher, F., et al. (2005). A dynamic balance between gene activation and repression regulates the shade avoidance response in Arabidopsis. Genes Dev. 19, 2811-2815. doi: $10.1101 / \operatorname{gad} .364005$

Shimono, M., Koga, H., Akagi, A., Hayashi, N., Goto, S., Sawada, M., et al. (2012). Rice WRKY45 plays important roles in fungal and bacterial disease resistance. Mol. Plant Pathol. 13, 83-94. doi: 10.1111/j.1364-3703.2011.00732.x

Splivallo, R., Fischer, U., Gobel, C., Feussner, I., and Karlovsky, P. (2009). Truffles regulate plant root morphogenesis via the production of auxin and ethylene. Plant Physiol. 150, 2018-2029. doi: 10.1104/pp.109.141325

Stepanova, A. N., Robertson-Hoyt, J., Yun, J., Benavente, L. M., Xie, D. Y., Dolezal, K., et al. (2008). TAA1-mediated auxin biosynthesis is essential for hormone crosstalk and plant development. Cell 133, 177-191. doi: 10.1016/j. cell.2008.01.047
Tsitsigiannis, D. I., and Keller, N. P. (2007). Oxylipins as developmental and hostfungal communication signals. Trends Microbiol. 15, 109-118. doi: 10.1016/j. tim.2007.01.005

van de Mortel, J. E., De Vos, R. C., Dekkers, E., Pineda, A., Guillod, L., Bouwmeester, K., et al. (2012). Metabolic and transcriptomic changes induced in Arabidopsis by the rhizobacterium Pseudomonas fluorescens SS101. Plant Physiol. 160, 2173-2188. doi: 10.1104/pp.112.207324

Conflict of Interest Statement: The authors declare that the research was conducted in the absence of any commercial or financial relationships that could be construed as a potential conflict of interest.

Copyright (C) 2017 Cordovez, Mommer, Moisan, Lucas-Barbosa, Pierik, Mumm, Carrion and Raaijmakers. This is an open-access article distributed under the terms of the Creative Commons Attribution License (CC BY). The use, distribution or reproduction in other forums is permitted, provided the original author(s) or licensor are credited and that the original publication in this journal is cited, in accordance with accepted academic practice. No use, distribution or reproduction is permitted which does not comply with these terms. 